\title{
A Derivative Matching Approach to Moment Closure for the Stochastic Logistic Model ${ }^{1}$
}

\author{
Abhyudai Singh and João Pedro Hespanha ${ }^{2}$ \\ Department of Electrical and Computer Engineering, University of California, Santa \\ Barbara, CA 93101.
}

\begin{abstract}
Continuous-time birth-death Markov processes serve as useful models in population biology. When the birth-death rates are nonlinear, the time evolution of the first $n$ order moments of the population is not closed, in the sense that it depends on moments of degree higher than $n$. For analysis purpose, the time evolution of the first $n$ order moments is often made to be closed by approximating these higher order moments as a nonlinear function of moments up to order $n$, which we refer to as the moment closure function.

In this paper, a systematic procedure for constructing moment closure functions of arbitrary order is presented for the stochastic logistic model. We obtain the moment closure function by first assuming a certain separable form for it, and then matching time derivatives of the exact (not closed) moment equations with that of the approximate (closed) equations for some initial time and set of initial conditions. The separable structure ensures that the steady-state solutions for the approximate equations are unique, real and positive, while the derivative matching guarantees a good approximation, at-least locally in time. Explicit formulas to construct these moment closure functions for arbitrary order of truncation $n$ are provided with higher values of $n$ leading to better approximation of the actual moment dynamics.

A host of other moment closure functions previously proposed in the literature are also investigated. Among these we show that only the ones that achieve derivative matching provide a close approximation to the exact solution. Moreover, we improve the accuracy of several previously proposed moment closure functions by forcing derivative matching.
\end{abstract}

Key words: Moment Closure, Stochastic Logistic Model, Stochastic Hybrid Systems

1 This material is based upon work supported by the Institute for Collaborative Biotechnologies through grant DAAD19-03-D-0004 from the U.S. Army Research Office and by the National Science Foundation under Grant No. CCR-0311084.

2 abhilengineering.ucsb.edu, hespanhalece.ucsb.edu 


\section{Introduction}

Continuous-time birth-death Markov processes have been extensively used for modeling stochasticity in population biology (Matis and Kiffe, 2002, 1996; Matis et al., 1998; Krishnarajah et al., 2005). The time evolution of these processes is typically described by a single equation for a probability function, where time and species populations appear as independent variables, called the Master or Kolmogorov equation (Bailey, 1964). Analytical solutions to the Master equations are generally not possible, however, solutions can be obtained numerically for small populations. For large populations, a more reasonable goal (and one that is of primary interest in applications) is to determine the time evolution of a few low-order statistical moments.

In this paper, a method for estimating low-order statistical moments is introduced for the stochastic logistic model. This model is the stochastic counterpart of the deterministic version of the logistic model (Verhulst, 1838; Pearl and Reed, 1920) and was first formulated by Feller (1939) as a continuous-time birth-death Markov process involving a single species, with birth and death rates being polynomials of degree 2. Although, one can directly use the Kolmogorov equation to derive differential equations for the time evolution of moments of the process, in this paper we use an alternative method. We model the stochastic logistic model as a Stochastic Hybrid System (SHS) whose state $\mathbf{x}$ is the population size of the species. Then, the time evolution of the moments is obtained using results from the SHS literature (e.g., Hespanha, 2004). Details of the stochastic logistic model and its modeling as a SHS are presented in Section 2.

It is well know that for the stochastic logistic model $\mathbf{x}=0$ is an absorbing state and eventual convergence to the origin is certain. For most biologically relevant problems one is typically interested in the distribution of the process conditioned on the event that absorption has not occurred. Let $P_{x}(t)=\mathrm{P}\{\mathbf{x}(t)=x \mid \mathbf{x}(t)>0\}$ denote the probability density function of the conditioned processes and $\mu_{m}(t)=\sum_{x=1}^{\infty} x^{m} P_{x}(t)$ its $m^{\text {th }}$ order uncentered moment. We show in Section 3 that the time derivative of the vector $\mu=\left[\mu_{1}, \ldots, \mu_{n}\right]^{T} \in \mathbb{R}^{n}$, where $n$ is the order of the truncation, is given by

$$
\dot{\mu}=\left(A+\lambda_{\text {ext }}(t) I\right) \mu+B \mu_{n+1}
$$

where $A$ and $B$ are appropriately defined matrices, $I$ is the identity matrix, and $\lambda_{\text {ext }}(t)$ an extinction rate. Assuming that the mean time to extinction is very large, the perturbation term $\lambda_{\text {ext }}(t) I$ is very small when compared to the matrix $A$ and can be ignored (Nåsell, 2003a). In spite of this, the dynamics of the above system is not closed, in the sense that the time evolution of the vector $\mu$ depends on the $n+1^{\text {th }}$ order moment $\mu_{n+1}$. We close the system by approximating $\mu_{n+1}$ as a nonlinear function $\varphi_{n+1}(\mu)$ of the moments up to order $n$. This procedure is commonly referred to as moment closure. We call $\varphi_{n+1}(\mu)$ the moment closure function for 
Table 1

Separable derivative-matching moment closure function $\varphi_{n+1}^{s}(\mu)$ for $n \in\{2,3,4\}$.

\begin{tabular}{cccc}
\hline & $n=2$ & $n=3$ & $n=4$ \\
\hline$\varphi_{n+1}^{s}(\mu)$ & $\frac{\mu_{2}^{3}}{\mu_{1}^{3}}$ & $\frac{\mu_{1}^{4} \mu_{3}^{4}}{\mu_{2}^{6}}$ & $\frac{\mu_{2}^{10} \mu_{4}^{5}}{\mu_{1}^{5} \mu_{3}^{10}}$ \\
\hline
\end{tabular}

$\mu_{n+1}$. Let $v=\left[v_{1}, \ldots, v_{n}\right]^{T}$ denote the state of the new closed system. Then, its dynamics is given by

$$
\dot{v}=A v+B \varphi_{n+1}(v)
$$

and is referred to as the truncated moment dynamics. We denote the states of (1) and (2) using different symbols because $\mu$ refers to the actual moment dynamics whereas $v$ to an approximated moment dynamics.

In Section 4, we consider moment closure functions which have the following separable form:

$$
\varphi_{n+1}^{s}(v)=v_{1}^{\gamma_{1}} v_{2}^{\gamma_{2}} \ldots v_{n}^{\gamma_{n}}
$$

for appropriately chosen constants $\gamma_{m} \in \mathbb{R}$. These constants are obtained by matching time derivatives of $\mu_{n+1}$ and $\varphi_{n+1}^{s}(v)$ in (1) and (2) respectively, at some initial time $t_{0}$ and initial condition $\mathbf{x}\left(t_{0}\right)=x_{0}$ with probability one. We refer to this moment closure as the Separable Derivative-Matching (SDM) moment closure. We show that for all $n \geq 2$, this determines the function $\varphi_{n+1}^{s}$ uniquely, which is independent of the birth and death rates. Table 1 shows the functions $\varphi_{n+1}^{s}$ that we obtained for truncations of degree $n=2,3$ and 4. The striking feature of the SDM moment closure is that the accuracy of the approximate moment dynamics improves by increasing the order of truncation and the dependence of higher order moments on lower order ones is consistent with $\mathbf{x}(t)$ being lognormally distributed, in spite of the fact that the derivative matching procedure used to construct $\varphi_{n+1}^{s}$ did not make any assumption on the distribution of the population.

Alternative moment closure methods that have appeared in literature typically construct the moment closure functions $\varphi$ by directly assuming the probability distribution to be normal (Whittle, 1957; Nåsell, 2003b), lognormal (Keeling, 2000), Poisson or binomial (Nåsell, 2003a). We refer to them as normal, lognormal, Poisson and binomial moment closures respectively and review them in Section 5. In Section 6, they are compared with the SDM moment closure based on how well the moment closure function $\varphi_{n+1}(\mu)$ approximates $\mu_{n+1}$. Towards that end, we introduce the error

$$
e_{n+1}(t):=\mu_{n+1}(t)-\varphi_{n+1}(\mu(t))=\sum_{i=0}^{\infty} \frac{\left(t-t_{0}\right)^{i}}{i !} \varepsilon_{n+1}^{i}\left(x_{0}\right)
$$


where we expanded the error as a Taylor series with $\varepsilon_{n+1}^{i}\left(x_{0}\right)$ defined to be

$$
\varepsilon_{n+1}^{i}\left(x_{0}\right):=\left.\frac{d^{i} \mu_{n+1}(t)}{d t^{i}}\right|_{t=t_{0}}-\left.\frac{d^{i} \varphi_{n+1}(\mu(t))}{d t^{i}}\right|_{t=t_{0}}
$$

We call $\varepsilon_{n+1}^{i}\left(x_{0}\right)$ the $i^{\text {th }}$ order derivative matching error. Ideally one would like this error to be zero but this is generally not possible. When $\mathbf{x}\left(t_{0}\right)=x_{0}$ with probability one, the derivative matching error is typically a polynomial in $x_{0}$. For example, for the SDM moment closure function the $0^{\text {th }}$ order derivative matching error is zero while for $i \geq 1$ the $i^{t h}$ order error is a polynomial in $x_{0}$ of degree $i+1$. Typically, the lesser the order of this polynomial, the lesser is the error $e_{n+1}(t)$, and hence the better is $\varphi_{n+1}(\mu)$ in approximating $\mu_{n+1}$.

We show that for $n=2$, all the above moment closure functions perform derivative matching except the Poisson moment closure function proposed by Nåsell (2003a). This is because, it has a $0^{\text {th }}$ order derivative matching error $\varepsilon_{3}^{0}\left(x_{0}\right)$ which grows linearly with $x_{0}$ while for SDM, lognormal, binomial and normal moment closure functions the $0^{\text {th }}$ order error is always zero. Hence, Nåsell's Poisson moment closure function exhibits a larger initial error than the others. We propose an alternative Poisson moment closure function, for which $\varepsilon_{3}^{0}\left(x_{0}\right)=0$, and show that it performs better than the one proposed by Nåsell (2003a).

Although the above moment closures provide good estimates for a second order of truncation $(n=2)$, it is typically beneficial to consider higher order of truncations because they lead to better moment approximations and reduce the errors by a few orders of magnitude. To the authors knowledge, moment closure for $n \geq 3$ has always been done in literature by assuming a normal distribution for the population (Nåsell, 2003b; Matis and Kiffe, 1996). We show that for $n=3$ the normal moment closure function also performs derivative matching with similar derivative matching error as for the SDM moment closure function, and hence, gives fairly good estimates of $\mu_{4}$. However, for $n=3$ we further propose a new moment closure function that yields lesser derivative matching errors when compared to separable derivative matching and normal moment closure functions, thus providing better estimates for $\mu_{4}$, at least locally in time.

In Section 7 we find the steady-state solutions of the truncated moment dynamics (2)-(3). We show that the separable structure of the SDM moment closure leads to analytical expressions for the approximate steady-state moments $v(\infty)$, which are always unique, real and positive for every order of truncation $n \geq 2$. In contrast, finding expressions for the steady-state moments using normal moment closure is typically done numerically for $n>2$, as this involves solving an $n^{\text {th }}$ degree polynomial and then identifying the biologically relevant steady-state among the $n$ roots of the polynomial (Matis and Kiffe, 1996).

Due to space limitations, the reader is sometimes referred to the technical report (Singh and Hespanha, 2006) for detailed derivations and extended discussions of 
some results.

\section{Stochastic Logistic Model}

\subsection{Model Formulation}

The stochastic logistic model is the stochastic birth-death analogous model of the well-known deterministic Verhulst-Pearl equations (Verhulst, 1838; Pearl and Reed, 1920; Pielou, 1977) and has been extensively used for modeling stochasticity in population biology (Matis and Kiffe, 2002, 1996; Matis et al., 1998; Krishnarajah et al., 2005). For this continuous-time birth-death Markov process, the conditional probabilities of a unit increase and decrease, respectively, in an "infinitesimal" time interval $(t, t+d t]$ are given by

$$
\begin{aligned}
& \mathrm{P}\{\mathbf{x}(t+d t)=x+1 \mid \mathbf{x}(t)=x\}= \begin{cases}\eta(x) d t, & \forall x \leq U \\
0, & \text { otherwise }\end{cases} \\
& \mathrm{P}\{\mathbf{x}(t+d t)=x-1 \mid \mathbf{x}(t)=x\}=\chi(x) d t,
\end{aligned}
$$

where $\mathbf{x}(t) \in \mathbb{N}$ represents the population size at time $t$,

$$
\eta(x):=a_{1} x-b_{1} x^{2}>0, \quad \chi(x):=a_{2} x+b_{2} x^{2}>0, \quad \forall x \in(0, U)
$$

and

$$
U:=a_{1} / b_{1} \in \mathbb{N}, \quad a_{1}>0, \quad a_{2}>0, \quad b_{1}>0, \quad b_{2} \geq 0 .
$$

We assume that the initial condition satisfies $\mathbf{x}\left(t_{0}\right) \in\{1,2, \ldots, U\}$, and hence, $\mathbf{x}(t) \in$ $\{0,1, \ldots, U\}, \forall t \in[0, \infty)$ with probability one. We call $U$ the population limit.

\subsection{Stationary and Quasi-Stationary Distributions}

Since the birth and death rates are zero for $x=0(\eta(0)=\chi(0)=0)$ we have that the state $\mathbf{x}=0$ is absorbing and eventual convergence to the origin is certain. However, it is common to use the stochastic logistic model mainly in the case where the mean time to extinction is very large. As the stationary distribution is degenerate with probability one at the origin, one is typically interested in the distribution of the process conditioned on the event that absorption has not occurred. In the sequel we denote by $\bar{P}_{x}(t)$ and $P_{x}(t)$ the probability density function of the unconditioned and conditioned processes, respectively. Thus for $x \in\{1, \ldots, U\}$ we have

$$
P_{x}(t)=\mathrm{P}\{\mathbf{x}(t)=x \mid \mathbf{x}(t)>0\}=\frac{\bar{P}_{x}(t)}{1-\bar{P}_{0}(t)}
$$


where $\bar{P}_{x}(t)=\mathrm{P}\{\mathbf{x}(t)=x\}$. The limit of $P_{x}(t)$ as $t \rightarrow \infty$ is known as the quasistationary distribution.

\subsection{Transient Distributions}

Using the Kolmogorov equations for $\bar{P}_{x}(t)$ one can show that $P_{x}(t)$ satisfies the following differential equations

$$
\begin{aligned}
& \dot{P}_{1}=\chi(2) P_{2}-[\eta(1)+\chi(1)] P_{1}+P_{1} \lambda_{\text {ext }}(t) \\
& \dot{P}_{x}=\chi(x+1) P_{x+1}-[\eta(x)+\chi(x)] P_{x}+\eta(x-1) P_{x-1}+P_{x} \lambda_{\text {ext }}(t), \\
& \quad \forall x \in\{2,3, \ldots, U-1\} \\
& \quad \vdots \\
& \dot{P}_{U}=-\chi(U) P_{U}+\eta(U-1) P_{U-1}+P_{U} \lambda_{\text {ext }}(t)
\end{aligned}
$$

where $\lambda_{\text {ext }}(t):=\chi(1) P_{1}(t)$ (Bailey, 1964; Nåsell, 2001). The variable $\lambda_{\text {ext }}(t)$ is an extinction rate in the sense that the conditional probability of extension in an "infinitesimal" interval $(t, t+d t]$ is given by

$$
\mathrm{P}\{\mathbf{x}(t+d t)=0 \mid \mathbf{x}(t)>0\}=\lambda_{\text {ext }}(t) d t .
$$

When the population limit $U$ is small, the above system of equations can be solved numerically. However, for large $U$, a more reasonable goal (and one that is of primary interest in applications) is to determine the evolution of the some lower-order moments of $P_{x}(t)$.

\section{Time Evolution of Moments}

\subsection{Modeling the Stochastic Logistic Model}

To model the time evolution of $\mathbf{x}(t)$, we consider a special class of systems known as Stochastic Hybrid Systems (SHS). These systems were introduced by Hespanha and Singh (2005) to model the stochastic time evolution of the populations of different species involved in a chemical reaction. More specifically, to fit the framework of our problem, these system are characterized by two reset maps:

$$
\mathbf{x} \mapsto \phi_{1}(\mathbf{x}):=\mathbf{x}+1, \quad \mathbf{x} \mapsto \phi_{2}(\mathbf{x}):=\mathbf{x}-1
$$

one corresponding to a birth and the other to a death, with associated transition intensities given by

$$
\lambda_{1}(\mathbf{x}):=\eta(\mathbf{x}), \quad \lambda_{2}(\mathbf{x}):=\chi(\mathbf{x})
$$


Between births and deaths the population remains constant and thus $\dot{\mathbf{x}}=0$. In essence, whenever a "birth event" or a "death event" takes place, the corresponding reset $\phi_{i}(\mathbf{x})$ is "activated" and $\mathbf{x}$ is reset accordingly, furthermore, the probability of the activation taking place in an "infinitesimal" time interval $(t, t+d t]$ is determined by the associated transition intensities $\lambda_{i}(\mathbf{x}) d t$.

\subsection{Moment Dynamics}

Given $m \in\{1,2, \ldots\}$, we define the $m^{\text {th }}$ order (uncentered) moment for both the unconditioned and conditioned process as

$$
\bar{\mu}_{m}(t)=\sum_{x=1}^{\infty} x^{m} \bar{P}_{x}(t):=\mathbf{E}\left[\mathbf{x}(t)^{m}\right], \quad \mu_{m}(t)=\sum_{x=1}^{\infty} x^{m} P_{x}(t), \quad \forall t \geq 0,
$$

respectively. The time evolution of $\bar{\mu}_{m}(t)$ is given by the following result, which is a straightforward application of results by Hespanha (Theorem 1, 2004) to the above SHS.

Theorem 1 The time evolution of $\bar{\mu}_{m}$ is given by

$$
\frac{d \bar{\mu}_{m}}{d t}=\mathbf{E}\left[\sum_{i=1}^{2}\left[\left(\phi_{i}(\mathbf{x})\right)^{m}-\mathbf{x}^{m}\right] \lambda_{i}(\mathbf{x})\right] .
$$

Using Theorem 1 (as in Appendix A, Singh and Hespanha, 2006) one can conclude that

$$
\dot{\bar{\mu}}_{m}=\sum_{p=1}^{2} \sum_{r=1}^{m+1} \mathrm{C}_{m+p-r}^{m} f(m+p-r, p) \bar{\mu}_{r}
$$

where we define $\mathrm{C}_{j}^{m}$ and $f(j, p)$ as follows ${ }^{3} \forall j, m, p \in \mathbb{N}$.

$$
\begin{aligned}
\mathrm{C}_{j}^{m}: & = \begin{cases}\frac{m !}{(m-j) ! j !} & m \geq j \geq 0 \\
0 & m<j\end{cases} \\
f(j, p) & := \begin{cases}0 & j=0 \\
a_{1}+(-1)^{j} a_{2} & j>0, p=1 \\
-b_{1}+(-1)^{j} b_{2} & j>0, p=2 .\end{cases}
\end{aligned}
$$

One can see from the right-hand-side of (10), that the time derivative of $\bar{\mu}_{m}$ is a linear combination of the moments $\bar{\mu}_{r}$, up to order $r=m+1$. Hence the time

$\bar{n} \mathrm{n}$ ! denotes the factorial of $\mathrm{n}$. 
evolution of the vector $\bar{\mu}=\left[\bar{\mu}_{1}, \bar{\mu}_{2}, \ldots, \bar{\mu}_{n}\right]^{T} \in \mathbb{R}^{n}$ is given by

$$
\dot{\bar{\mu}}=A \bar{\mu}+B \bar{\mu}_{n+1}
$$

for some $n \times n$ and $n \times 1$ matrices $A$ and $B$ which have the following structure

$$
A=\left[\begin{array}{ccccc}
* * & 0 & 0 & \ldots & 0 \\
* * & * & 0 & \ldots & 0 \\
\vdots & \vdots & \ddots & \ddots & \vdots \\
* * & * & * & * & * \\
* * & * & * & * & *
\end{array}\right], \quad B=\left[\begin{array}{c}
0 \\
0 \\
\vdots \\
0 \\
*
\end{array}\right]
$$

where each $*$ denotes a possibly non-zero entry. From (5), (9) we have

$$
\mu_{m}(t)=\frac{\bar{\mu}_{m}(t)}{1-\bar{P}_{0}(t)}
$$

which using (13) and $\dot{\bar{P}}_{0}(t)=\chi(1) \bar{P}_{1}(t)$ leads to

$$
\dot{\mu}=\left(A+\lambda_{\text {ext }}(t) I\right) \mu+B \mu_{n+1},
$$

where $\mu=\left[\mu_{1}, \mu_{2}, \ldots, \mu_{n}\right]^{T} \in \mathbb{R}^{n}$ and $\lambda_{\text {ext }}(t):=\chi(1) P_{1}(t)$ is the extinction rate. The dynamics of this system is not closed because the time-derivative of the vector $\mu$ depends both on the $n+1^{\text {th }}$ order moment $\mu_{n+1}$ and on the extinction rate $\lambda_{\text {ext }}$, which are not part of the state $\mu$. However, when the mean time to extinction is very large, the perturbation term $\lambda_{\text {ext }}(t) I$ is very small when compared to the matrix $A$ and can be ignored (Nåsell, 2003a). Our goal now is to close the dynamics of (16) by approximating $\mu_{n+1}$ as a nonlinear function of $\mu$ given by $\varphi_{n+1}(\mu)$. This gives the closed approximate moment dynamics

$$
\dot{v}=A v+B \varphi_{n+1}(v), \quad v=\left[v_{1}, v_{2}, \ldots, v_{n}\right]^{T} .
$$

We call (17) the truncated moment dynamics and $\varphi_{n+1}(\mu)$ the moment closure function for $\mu_{n+1}$.

When a sufficiently large but finite number of derivatives of $\mu(t)$ and $v(t)$ match point-wise, then, the difference between solutions to (16) and (17) remains close on a given compact time interval. This follows from a Taylor series approximation argument. To be more precise, for each $\delta>0$ and integer $N$, there exists a time $T>t_{0}$, for which the following result holds: Assume that for every $t_{0} \geq 0$,

$$
\mu\left(t_{0}\right)=v\left(t_{0}\right) \quad \text { and }\left.\quad \frac{d^{i} \mu(t)}{d t^{i}}\right|_{t=t_{0}}=\left.\frac{d^{i} v(t)}{d t^{i}}\right|_{t=t_{0}}, \forall i \in\{1, \ldots, N\}
$$


where $\frac{d^{i} \mu(t)}{d t^{i}}$ and $\frac{d^{i} v(t)}{d t^{i}}$ represent the $i^{t h}$ time derivative of $\mu(t)$ and $v(t)$ along the trajectories of system (16) and (17) respectively. Then,

$$
\|\mu(t)-v(t)\| \leq \delta, \quad \forall t \in\left[t_{0}, T\right] .
$$

In the next section we use (18) to construct moment closure functions $\varphi_{n+1}(\mu)$.

\section{Separable Derivative-Matching Moment Closures}

In this section we construct truncated moment dynamics (17) for the stochastic logistic model using (18). After replacing (16) and (17) in (18), the equality (18) becomes a PDE on $\varphi_{n+1}$. We will seek for solutions $\varphi_{n+1}(\mu)$ to this PDE that have the following separable form

$$
\varphi_{n+1}^{s}(\mu)=\prod_{m=1}^{n} \mu_{m}^{\gamma_{m}}
$$

for appropriately chosen constants $\gamma_{m} \in \mathbb{R}$. In the sequel we refer to such $\varphi_{n+1}^{s}(\mu)$ as a Separable Derivative-Matching (SDM) moment closure function for $\mu_{n+1}$.

One can see that the infinite vector $\mu_{\infty}=\left[\mu_{1}, \mu_{2}, \cdots\right]^{T} \in \Omega_{\infty}$ can be expressed as

$$
\mu_{\infty}(t)=\sum_{x=1}^{\infty}\left[\begin{array}{c}
x \\
x^{2} \\
x^{3} \\
\vdots
\end{array}\right] P_{x}(t) .
$$

Hence, the infinite vectors $\left[x, x^{2}, x^{3}, \ldots\right]^{T}$, which corresponds to a degenerate distribution $\mathbf{x}(t)=x$ with probability one, form a natural basis for $\Omega_{\infty}$. In particular, we will find constants $\gamma_{m}$ that satisfy (18) for each initial vector $\mu_{\infty}\left(t_{0}\right)$ belonging to this basis, i.e. for the class of deterministic initial conditions. Also note from (6) that the $i^{t h}$ time derivative of $\lambda_{\text {ext }}(t):=\chi(1) P_{1}(t)$ is a function of $P_{1}(t), P_{2}(t), \ldots$ $P_{i+1}(t)$. Thus for $\mathbf{x}\left(t_{0}\right)=x_{0}$ with probability one, we have that

$$
\left.\frac{d^{i}\left(\lambda_{\mathrm{ext}}(t) \mu(t)\right)}{d t^{i}}\right|_{t=t_{0}}=0, \quad \forall x_{0} \geq i+2
$$

and hence, these terms do not contribute when we calculate the $i$ th time derivatives of $\mu(t)$ using (16).

Generally it is not possible to find $\gamma_{m}$ for which (18) holds exactly. We will therefore 
relax this condition and simply demand the following

$$
\mu\left(t_{0}\right)=v\left(t_{0}\right) \quad \text { and }\left.\quad \frac{d^{i} \mu(t)}{d t^{i}}\right|_{t=t_{0}}=\left.\frac{d^{i} v(t)}{d t^{i}}\right|_{t=t_{0}}+\mathbf{E}\left[\varepsilon_{i}\left(\mathbf{x}\left(t_{0}\right)\right)\right]
$$

$\forall i \in\{1,2, \ldots\}$, where each element of the vector $\varepsilon_{i}\left(\mathbf{x}\left(t_{0}\right)\right)$ is a polynomial in $\mathbf{x}\left(t_{0}\right)$. One can think of (21) as an approximation to (18) that is valid as long as $\left.\frac{d^{i} \mu(t)}{d t^{i}}\right|_{t=t_{0}}$ dominates $\mathbf{E}\left[\varepsilon_{i}\left(\mathbf{x}\left(t_{0}\right)\right)\right]$. The following theorem summarizes the main result (see proof in Appendix B, Singh and Hespanha, 2006).

Theorem 2 Let $\gamma_{m}, m \in\{1, \ldots, n\}$ be chosen as

$$
\gamma_{m}=(-1)^{n-m} \mathrm{C}_{m}^{n+1}
$$

Then, for every deterministic initial condition $v\left(t_{0}\right)=\mu\left(t_{0}\right)=\left[x_{0}, x_{0}^{2}, \ldots, x_{0}^{n}\right]^{T}, x_{0} \geq$ $n+1$ that corresponds to $\mathbf{x}\left(t_{0}\right)=x_{0}$ with probability one, we have that

$$
\begin{aligned}
\left.\frac{d \mu(t)}{d t}\right|_{t=t_{0}} & =\left.\frac{d v(t)}{d t}\right|_{t=t_{0}} \\
\left.\frac{d^{2} \mu(t)}{d t^{2}}\right|_{t=t_{0}} & =\left.\frac{d^{2} v(t)}{d t^{2}}\right|_{t=t_{0}}+\varepsilon_{2}\left(x_{0}\right),
\end{aligned}
$$

where $\frac{d^{i} \mu}{d t^{i}}$ and $\frac{d^{i} v}{d t^{i}}$ denote the $i^{\text {th }}$ time derivative of $\mu$ and $v$ along the trajectories of the systems (16) and (17), respectively, and the $n^{\text {th }}$ element of the vector $\varepsilon_{2}\left(x_{0}\right)$ is a polynomial in $x_{0}$ of degree 2 with all other elements being zero.

Remark 1 Using (10) and (15) it can be shown that $\frac{d^{2} \mu_{n}(t)}{d t^{2}}$ is a linear combination of moments of $\mathbf{x}$ up to order $n+2$ and therefore $\left.\frac{d^{2} \mu_{n}(t)}{d t^{2}}\right|_{t=t_{0}}$ is a polynomial in $x_{0}$ of degree $n+2$. We thus conclude from Theorem 2 that 4

$$
\frac{\varepsilon_{2}^{n}\left(x_{0}\right)}{\left.\frac{d^{2} \mu_{n}(t)}{d t^{2}}\right|_{t=t_{0}}}=O\left(x_{0}^{-n}\right)
$$

where $\varepsilon_{2}^{n}\left(x_{0}\right)$ is the $n^{\text {th }}$ element of $\varepsilon_{2}\left(x_{0}\right)$. This shows that the term $\left.\frac{d^{2} \mu_{n}(t)}{d t^{2}}\right|_{t=t_{0}}$ dominates $\varepsilon_{2}^{n}\left(x_{0}\right)$ by $x_{0}^{-n}$.

Remark 2 It can be verified that the SDM moment closure function also matches derivatives of degree higher than 2 in (23) with small errors. For example for $n \in$ $\{2,3,4\}$ and $i \in\{2, \ldots, 9\}$, we have

$$
\left.\frac{d \mu(t)}{d t}\right|_{t=t_{0}}=\left.\frac{d v(t)}{d t}\right|_{t=t_{0}}
$$

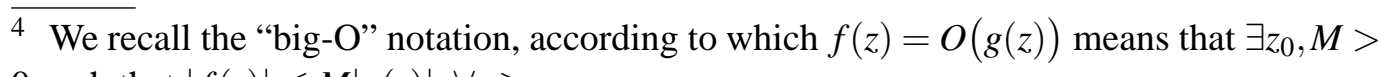
0 such that $|f(z)| \leq M|g(z)|, \forall z \geq z_{0}$. 


$$
\left.\frac{d^{i} \mu(t)}{d t^{i}}\right|_{t=t_{0}}=\left.\frac{d^{i} v(t)}{d t^{i}}\right|_{t=t_{0}}+\varepsilon_{i}\left(x_{0}\right)
$$

and as in (24) the elements of vector $\left.\frac{d^{i} \mu(t)}{d t^{i}}\right|_{t=t_{0}}$ dominate the corresponding elements of $\varepsilon_{i}\left(x_{0}\right)$ by $x_{0}^{-n}$. We conjecture that the above equality holds $\forall n \in \mathbb{N}$ and $\forall i \in \mathbb{N}$ but we only verified it for $n$ up to 4 and $i$ up to 9 . Hence, with increasing $n$, the truncated moment dynamics $v(t)$ should provide a more accurate approximations to the lower order moments $\mu(t)$.

Remark 3 Table 1 shows the functions $\varphi_{n+1}^{s}$ corresponding to $\gamma_{m}$ chosen according to (22) for $n=2,3$ and 4. The dependence of $\mu_{n+1}$ on lower order moments $\mu_{1}, \ldots, \mu_{n}$ as given by the SDM moment closure function, is consistent with $\mathbf{x}(t)$ being lognormally distributed (see Example 3, Section 5, Singh and Hespanha, 2006, for details).

\section{Distribution-Based Moment Closures}

Most moment closure techniques that appeared in the literature start by assuming a specific distributions for the population, and use this assumption to express higher order moments as a function of the lower order ones. This has been done for well known classes of distributions, such as normal (Whittle, 1957; Matis and Kiffe, 1996; Nåsell, 2003b), lognormal (Keeling, 2000), Poisson and binomial (Nåsell, 2003a) and for these distribution we simply say that $\varphi_{n+1}$ is the normal, lognormal, Poisson, or binomial moment closure function.

For a second order truncation $(n=2)$, Table 2 lists several moment closure functions for $\mu_{3}$, including the SDM. Nåsell-Poisson refers to the Poisson moment closure function proposed by Nåsell (2003a), while new-Poisson refers an alternative Poisson moment closure function that we propose, which, as we will see in the next section, performs better than the one proposed by Nåsell (2003a). The explanation for this lies in the fact that the new-Poisson has better derivative matching properties than the Nåsell-Poisson, in the sense of (21). In the sequel we use superscripts $s$, $l, g, p 1, p 2$ and $b$ to denote SDM, lognormal, normal, Nåsell-Poisson, new-Poisson and binomial moment closure functions, respectively. In most of the literature, moment closure for orders of truncation higher than $2(n \geq 3)$, is typically done by assuming a normal distribution for the population (Matis and Kiffe, 1996; Nåsell, 2003a). Then, using the property of the normal distribution that all $3^{\text {rd }}$ and higher order cumulants are zero, one obtains a normal moment closure function by setting the $n+1^{\text {th }}$ order cumulant equal to zero. We recall that the third and forth order cumulants can be expressed in terms of the uncentered moments as follows:

$$
\kappa_{3}=\mu_{3}-3 \mu_{1} \mu_{2}+2 \mu_{1}^{3}, \quad \kappa_{4}=\mu_{4}-4 \mu_{1} \mu_{3}-3 \mu_{2}^{2}+12 \mu_{2} \mu_{1}^{2}-6 \mu_{1}^{4} .
$$


Table 2

Moment closure functions for second order truncation $(n=2)$, corresponding to the different moment closure techniques discussed in this paper.

\begin{tabular}{ll}
\hline Moment Closure Technique & \multicolumn{1}{c}{ Moment Closure Functions } \\
\hline SDM & $\varphi_{3}^{s}(\mu)=\frac{\mu_{2}^{3}}{\mu_{1}^{3}}$ \\
Normal & $\varphi_{3}^{g}(\mu)=3 \mu_{2} \mu_{1}-2 \mu_{1}^{3}$ \\
Lognormal & $\varphi_{3}^{l}(\mu)=\frac{\mu_{2}^{3}}{\mu_{1}^{3}}$ \\
Nåsell-Poisson & $\varphi_{3}^{p 1}(\mu)=\mu_{1}+3 \mu_{1} \mu_{2}-2 \mu_{1}^{3}$ \\
New-Poisson & $\varphi_{3}^{p 2}(\mu)=\mu_{2}-\mu_{1}^{2}+3 \mu_{1} \mu_{2}-2 \mu_{1}^{3}$ \\
Binomial & $\varphi_{3}^{b}(\mu)=2 \frac{\left(\mu_{2}-\mu_{1}^{2}\right)^{2}}{\mu_{1}}-\left(\mu_{2}-\mu_{1}^{2}\right)+3 \mu_{1} \mu_{2}-2 \mu_{1}^{3}$ \\
\hline
\end{tabular}

For $n=3$, this leads to the following normal moment closure functions for $\mu_{4}$

$$
\varphi_{4}^{g}(\mu)=4 \mu_{1} \mu_{3}+3 \mu_{2}^{2}-12 \mu_{2} \mu_{1}^{2}+6 \mu_{1}^{4}
$$

The reader is referred to Singh and Hespanha (2006) for the derivations of the above moment closure functions and a detailed discussion on distribution-based moment closures.

\section{Comparison of Transient Performance of Moment Closures}

In this section, we compare the transient performance of different moment closure techniques using the error

$$
e_{n+1}(t):=\mu_{n+1}(t)-\varphi_{n+1}(\mu(t))=\sum_{i=0}^{\infty} \frac{\left(t-t_{0}\right)^{i}}{i !} \varepsilon_{n+1}^{i}\left(x_{0}\right)
$$

where

$$
\varepsilon_{n+1}^{i}\left(x_{0}\right):=\left.\frac{d^{i} \mu_{n+1}(t)}{d t^{i}}\right|_{t=t_{0}}-\left.\frac{d^{i} \varphi_{n+1}(\mu(t))}{d t^{i}}\right|_{t=t_{0}}
$$

We call $\varepsilon_{n+1}^{i}\left(x_{0}\right)$ the derivative matching error. Ideally, one would like to have $\varepsilon_{n+1}^{i}\left(x_{0}\right)=0$, but as already pointed out in Section 4 this is generally not possible. With deterministic initial conditions $\mu_{\infty}\left(t_{0}\right)=\left[x_{0}, x_{0}^{2}, x_{0}^{3}, \ldots\right]^{T}$, as in Theorem 2 , the derivative matching error is typically a polynomial in $x_{0}$. The lesser the order of this polynomial, the better is $\varphi_{n+1}(\mu)$ in approximating $\mu_{n+1}$. 


\subsection{Moment Closures for $n=2$}

We recall from Table 2 that $\varphi_{3}^{l}(\mu)=\varphi_{3}^{s}(\mu)$, and therefore we do not need to discuss lognormal moment closure separately. By substituting $\varphi_{3}^{s}(\mu), \varphi_{3}^{g}(\mu), \varphi_{3}^{p 1}(\mu)$, $\varphi_{3}^{p 2}(\mu)$ and $\varphi_{3}^{b}(\mu)$ from Table 2 in (26)-(27), one obtains the corresponding derivative matching errors, which will be denoted using the appropriate superscripts.

Using Table 2 and symbolic manipulation in Mathematica, we can show that

$$
\begin{aligned}
{ }^{s} \varepsilon_{3}^{0}\left(x_{0}\right) & ={ }^{g} \varepsilon_{3}^{0}\left(x_{0}\right)={ }^{2} \varepsilon_{3}^{0}\left(x_{0}\right)={ }^{b} \varepsilon_{3}^{0}\left(x_{0}\right)=0 \\
{ }^{p 1} \varepsilon_{3}^{0}\left(x_{0}\right) & =-x_{0} . \\
{ }^{*} \varepsilon_{3}^{i}\left(x_{0}\right) & \in P_{x_{0}}(i+1), *=\{s, g, p 1, p 2, b\}, \quad \forall i \in\{1,2, \ldots\}
\end{aligned}
$$

where $P_{x_{0}}(j)$ denotes the set of polynomials in $x_{0}$ of degree $j$. Since ${ }^{p 1} \varepsilon_{3}^{0}\left(x_{0}\right)=-x_{0}$, the Nåsell-Poisson moment closure function will have a large initial error, especially for large initial conditions, when compared to all other moment closure functions. For all $i \in\{1,2, \ldots\}$, all of these moment closure functions match derivatives, with the derivative matching error being of the same order in $x_{0}$. The simulation results discussed below show that with the exception of Nåsell-Poisson moment closure function, which consistently provides the worst estimates, all other moment closure functions perform fairly well.

Example: We consider the stochastic logistic model with

$$
a_{1}=.30, a_{2}=.02, b_{1}=.015, b_{2}=.001,
$$

which is used by Matis et al. (1998) to model the population dynamics of the African Honey Bee. Using (17) with the matrices $A$ and $B$ computed in (10), we have the following truncated moment dynamics

$$
\left[\begin{array}{c}
\dot{v}_{1} \\
\dot{v}_{2}
\end{array}\right]=\left[\begin{array}{cc}
0.28 & -0.016 \\
.32 & .546
\end{array}\right]\left[\begin{array}{c}
v_{1} \\
v_{2}
\end{array}\right]-\left[\begin{array}{c}
0 \\
0.032
\end{array}\right] \varphi_{3}(v) .
$$

The time evolution of the moments corresponding to different moment closure techniques is obtained by substituting the appropriate moment closure function from Table 2 in place of $\varphi_{3}(v)$. In order to evaluate the performance of these moment closure functions for all time, we compute the exact evolution of the moments $\mu(t)$. This is only possible because the population limit $U=25$ is small and one can obtain the exact solution by numerically solving the equation (6). Figure 1 and 2 contains plots of the mean and variance errors, respectively, for the different moment closure functions with $x_{0}=5$ and $x_{0}=20$. For $x_{0}=20$ the binomial moment closure function provides the best estimate both initially and at steady-state, whereas for $x_{0}=5$ the new-Poisson moment closure function does best initially, but the 

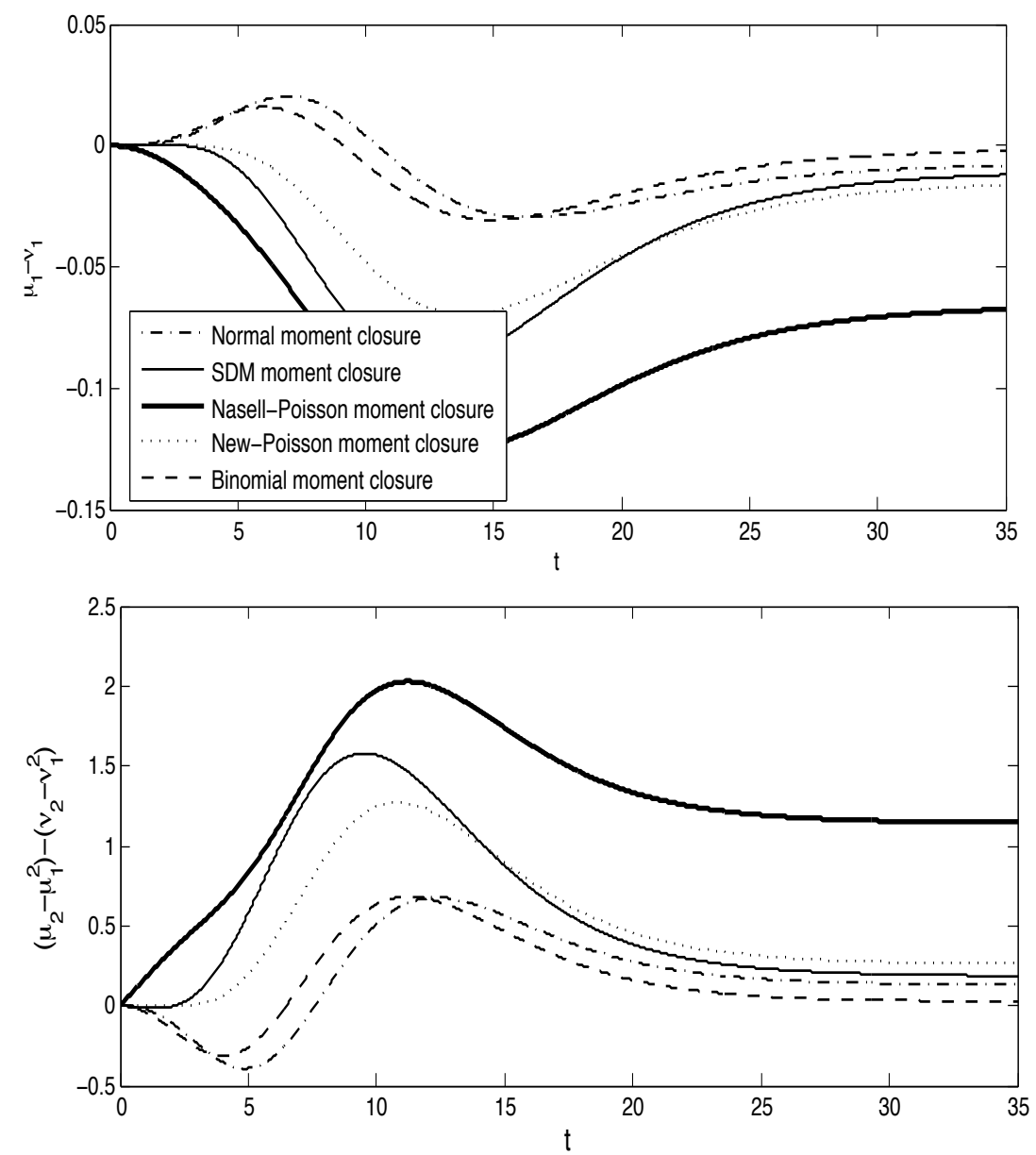

Fig. 1. Evolutions of the mean error $\mu_{1}-v_{1}$ and of the variance error $\left(\mu_{2}-\mu_{1}^{2}\right)-\left(v_{2}-v_{1}^{2}\right)$ for the different moment closure functions in Table 2 for $n=2$, with parameters as in (29) and $x_{0}=5$.

binomial moment closure function continues to provide the most accurate steadystate estimate. As one would expect from (28), the Nåsell-Poisson moment closure function performs the worst.

\subsection{Moment Closures for $n=3$}

In this section, we propose a new moment closure function given by

$$
\varphi_{4}^{z}(\mu)=4 \mu_{1} \mu_{3}+3 \mu_{2}^{2}-12 \mu_{2} \mu_{1}^{2}+6 \mu_{1}^{4}+\mu_{2}-\mu_{1}^{2}
$$

and refer to it as the Zero first-order error moment closure function. For comparison purpose we recall from Table 1 and Section 5 that the SDM and normal moment 

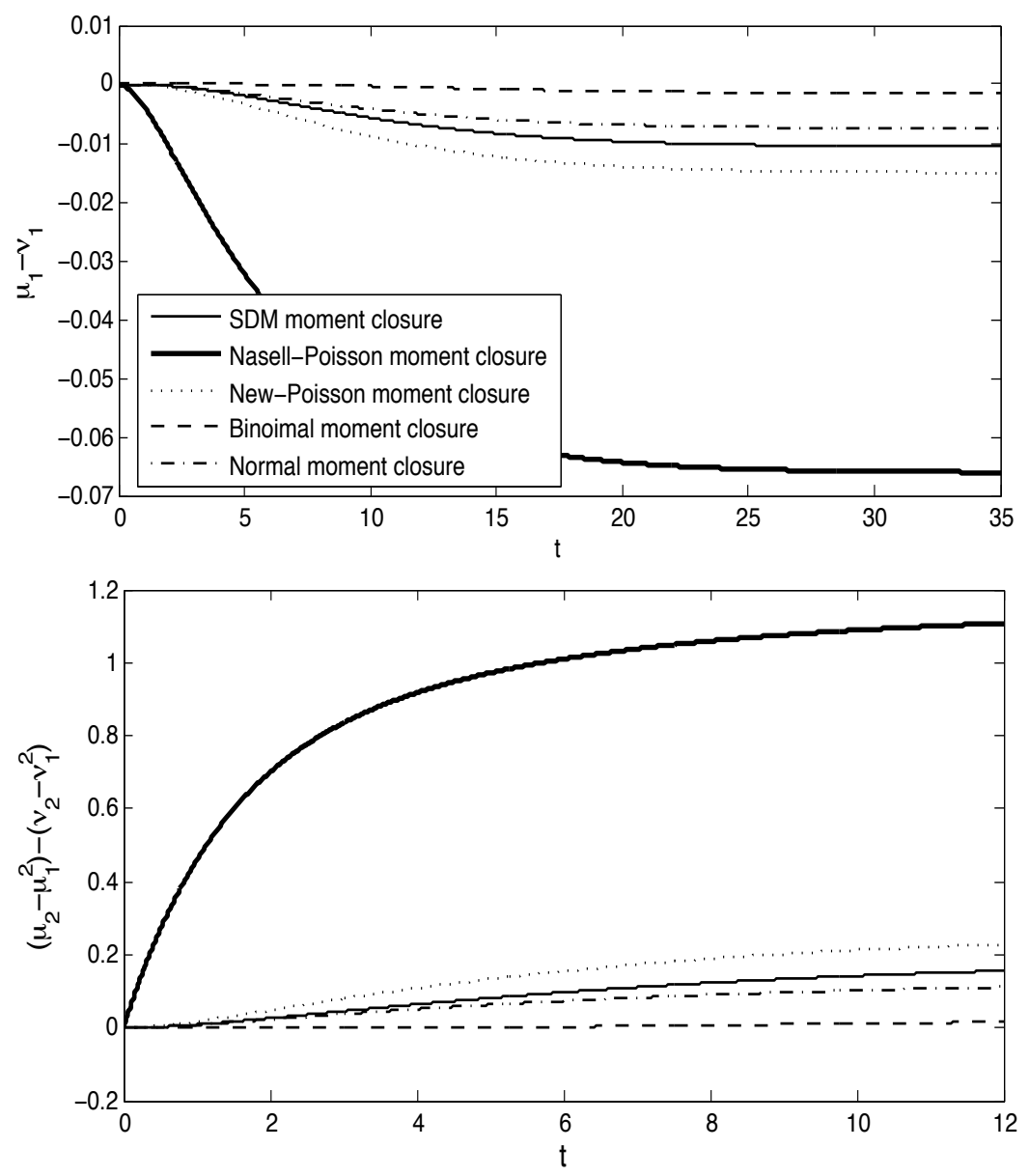

Fig. 2. Evolutions of the mean error $\mu_{1}-v_{1}$ and of the variance error $\left(\mu_{2}-\mu_{1}^{2}\right)-\left(v_{2}-v_{1}^{2}\right)$ for the different moment closure functions in Table 2 for $n=2$, with parameters as in (29) and $x_{0}=20$.

closure functions for $n=3$ are given by

$$
\begin{aligned}
\varphi_{4}^{s}(\mu) & =\frac{\mu_{1}^{4} \mu_{3}^{4}}{\mu_{2}^{6}} \\
\varphi_{4}^{g}(\mu) & =4 \mu_{1} \mu_{3}+3 \mu_{2}^{2}-12 \mu_{2} \mu_{1}^{2}+6 \mu_{1}^{4},
\end{aligned}
$$

respectively. The above moment closure functions yield the following derivative matching errors:

$$
\begin{aligned}
& { }^{*} \varepsilon_{4}^{0}\left(x_{0}\right)=0, \quad *=\{g, s, z\} \\
& { }^{z} \varepsilon_{4}^{1}\left(x_{0}\right)=0, \quad{ }^{\dagger} \varepsilon_{4}^{1}\left(x_{0}\right) \in P_{x_{0}}(2), \dagger=\{g, s\} \\
& { }^{*} \varepsilon_{4}^{i}\left(x_{0}\right) \in P_{x_{0}}(i+1), \quad *=\{g, s, z\}, \quad \forall i \in\{1,2, \ldots\} .
\end{aligned}
$$

¿From (33) one can see the following:

- The normal moment closure function also performs derivative-matching yield- 

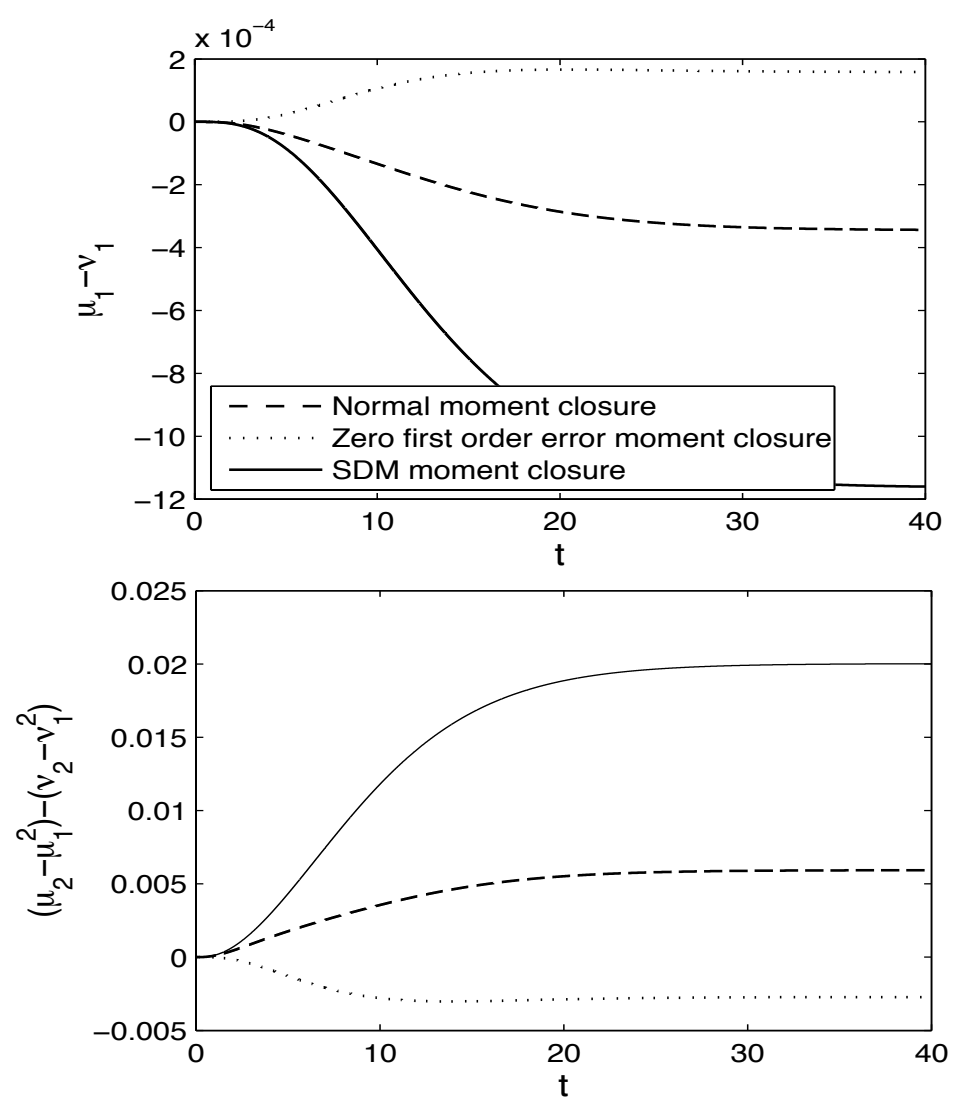

Fig. 3. Evolutions of the mean error $\mu_{1}-v_{1}$, variance error $\left(\mu_{2}-\mu_{1}^{2}\right)-\left(v_{2}-v_{1}^{2}\right)$ for the different moment closure functions (30)-(32) for $n=3$, with parameters as in (29) and $x_{0}=20$.

ing the same order of derivative matching error as the SDM moment closure function, and hence, provides reasonably good estimates for $\mu_{4}$.

- Unlike the other moment closure functions, the zero first-order error moment closure function yields zero $0^{\text {th }}$ and $1^{\text {st }}$ order derivative matching error, and hence, provides the best estimates for $\mu_{4}$, at least near $t=0$.

In order to confirm our predictions above that were based on the expressions (33), we consider the stochastic logistic model with parameters as in (29), $n=3$ and $x_{0}=20$. Using (10), we have the following truncated moment dynamics

$$
\left[\begin{array}{c}
\dot{v}_{1} \\
\dot{v}_{2} \\
\dot{v}_{3}
\end{array}\right]=\left[\begin{array}{ccc}
0.28 & -0.016 & 0 \\
.32 & .546 & -0.032 \\
.28 & .944 & .798
\end{array}\right]\left[\begin{array}{c}
v_{1} \\
v_{2} \\
v_{3}
\end{array}\right]-\left[\begin{array}{c}
0 \\
0 \\
0.048
\end{array}\right] \varphi_{3}(v) .
$$

Substituting the moment closer functions (30)-(32) in place of $\varphi_{3}(v)$, we obtain the corresponding approximate time evolution of moments. Figure 3 contains plots of the mean and variance errors. As expected both the normal and SDM moment closure functions provide good estimates. One can also see that the zero first-order 
error moment closure function (30), which guarantees the best approximation near $t=0$ actually provides in this case the most accurate estimate for $\mu_{4}$ for all time.

Comparing the plots in Figures 1 and 3, we observe that the mean and variance errors for $x_{0}=20$ obtained with a third order truncation $(n=3)$ are an order of magnitude smaller than the ones obtained with a second order truncation $(n=2)$.

\section{Steady-State Solutions of the Truncated Moment Dynamics}

We now show that the SDM moment closure leads to a unique positive equilibrium for the truncated dynamics (17) and provide analytical expressions for this equilibrium.

Consider the truncated moment dynamics of degree $n \geq 2$ with the moment closure functions given in Table 1. From (17), the steady-state solution $v(\infty)$ can be computed by solving

$$
0=A v(\infty)+B \varphi_{n+1}^{s}(v(\infty))
$$

where the coefficient of the matrices $A, B$ can be deduced from (10). Solving these equations, we obtain

$$
\begin{aligned}
v_{2}(\infty) & =c_{1} v_{1}(\infty) \\
\vdots & \\
v_{n}(\infty) & =c_{n-1} v_{1}(\infty) \\
\varphi_{n+1}^{s}(v(\infty)) & =c_{n} v_{1}(\infty)
\end{aligned}
$$

for appropriate positive real numbers $c_{1}, \ldots, c_{n}$. In terms of the parameters $a_{1}, b_{1}$, $a_{2}$ and $b_{2}$, the first three constants $c_{1}, c_{2}, c_{3}$ are given by the following expressions

$$
\begin{aligned}
& c_{1}=K, \quad c_{2}=K^{2}+\sigma^{2}, \quad c_{3}=K^{3}+3 K \sigma^{2}+\bar{\sigma} \sigma^{2} \\
& K=\frac{a_{1}-a_{2}}{b_{1}+b_{2}}, \quad \sigma^{2}=\frac{a_{1} b_{2}+b_{1} a_{2}}{\left(b_{1}+b_{2}\right)^{2}}, \quad \bar{\sigma}=\frac{b_{2}-b_{1}}{b_{2}+b_{1}} \text {. }
\end{aligned}
$$

¿From (35) and Table 1 we obtain the following steady-state values for $\varphi_{n+1}^{s}(v(\infty))$, $n \in\{2,3,4, \ldots\}$ :

$$
\varphi_{3}^{s}(v(\infty))=c_{1}^{3}, \quad \varphi_{4}^{s}(v(\infty))=\frac{c_{2}^{4}}{c_{1}^{6}} v_{1}(\infty)^{2}, \quad \varphi_{5}^{s}(v(\infty))=\frac{c_{1}^{10} c_{3}^{5}}{c_{2}^{10}}
$$

Substituting (36) in (35) yields the following unique non-trivial solutions for the 
steady-state mean $v_{1}(\infty)$

$$
v_{1}(\infty)= \begin{cases}\frac{c_{1}^{3}}{c_{2}}=\frac{K}{1+\frac{\sigma^{2}}{K^{2}}} & n=2 \\ \frac{c_{1}^{6} c_{3}}{c_{2}^{4}}=\frac{K\left(1+\frac{3 \sigma^{2}}{K^{2}}+\frac{\bar{\sigma} \sigma^{2}}{K^{3}}\right)}{\left(1+\frac{\sigma^{2}}{K^{2}}\right)^{4}} & n=3 \\ \frac{c_{1}^{10} c_{3}^{5}}{c_{2}^{10} c_{4}} & n=4 .\end{cases}
$$

The corresponding high-order uncentered moments $v_{2}(\infty), \ldots, v_{n}(\infty)$ can be calculated from (35). We conclude that the SDM moment closure function always yields a unique non-trivial positive, real, steady-state for every truncation order $n \geq 2$. Moreover, the separable structure of the SDM moment closure leads to analytical expressions for the steady-state moments. In contrast, finding the steady-state moments for the normal moment closure requires solving an $n^{\text {th }}$ degree polynomial in $v_{1}(\infty)$ and then identifying the biologically relevant steady-state among the $n$ roots of the polynomial. For $n>2$ this can generally only be done numerically and one does not obtain analytic expressions for the steady-state moments (Matis and Kiffe, 1996).

\section{Conclusion and Future Work}

A procedure for constructing moment closures for the stochastic logistic model was presented. This was done by first assuming a separable form for the moment closure function $\varphi_{n+1}(v)$, and then, matching its time derivatives with $\mu_{n+1}$, at some initial time $t_{0}$ for a basis of initial conditions $x\left(t_{0}\right)=x_{0}$. We showed that there exists a unique separable derivative-matching moment closure function for which the $i^{\text {th }}$ order derivative matching error is a polynomial in $x_{0}$ of degree $i+1$ for all $i \in$ $\{1,2, \ldots\}$ and zero for $i=0$. Explicit formulas to construct these moment closure functions for arbitrary order of truncation $n$ were provided with higher values of $n$ leading to better approximation of the actual moment dynamics.

The separable structure of this moment closure greatly simplified the process of finding the steady-state of the truncated moment dynamics which were always unique, real and positive. Comparisons with alternative moment closure techniques available in literature illustrated how derivative matching can be used as a effective tool for gauging the performance of moment closure functions. We showed that for $n=2$, with the exception of the Nåsell-Poisson, all other moment closure functions in Table 2 perform derivative matching and provide fairly good estimates for $\mu_{3}$. For $n=3$, a new zero first-order error moment closure function was also proposed, guaranteeing better approximations, at least locally in time, as compared to the other moment closure techniques discussed in this paper. 
The truncated moment dynamics presented in this paper only capture the quasistationary distribution and do not provide information about the time taken to reach extinction. Finding alternative moment closure techniques that provide information about extinction is a subject for future research. Another direction for future research is the extension of the results in this paper to multi-species birth-death Markov processes. Primary results on this topic can be found in Hespanha and Singh (2005).

\section{References}

Bailey, N. T. J., 1964. The Elements of Stochastic Processes. Wiley, New York.

Feller, W., 1939. Die grundlagen der volterraschen theorie des kampfes ums dasein in wahrscheinlichkeitstheoretischer behandlung. Acta Biotheoretica 5, 11-40.

Hespanha, J. P., Mar. 2004. Stochastic hybrid systems: Applications to communication networks. In: Alur, R., Pappas, G. J. (Eds.), Hybrid Systems: Computation and Control. No. 2993 in Lect. Notes in Comput. Science. Springer-Verlag, Berlin, pp. 387-401.

Hespanha, J. P., Singh, A., 2005. Stochastic models for chemically reacting systems using polynomial stochastic hybrid systems. Int. J. of Robust and Nonlinear Control 15, 669-689.

Keeling, M. J., 2000. Multiplicative moments and measures of persistence in ecology. J. of Theoretical Biology 205, 269-281.

Krishnarajah, I., Cook, A., Marion, G., Gibson, G., 2005. Novel moment closure approximations in stochastic epidemics. Bull. Math. Bio. 67, 855-873.

Matis, J. H., Kiffe, T. R., 1996. On apprroximating the moments of the equilibrium distribution of a stochastic logisitc model. Biometrics 52, 980-991.

Matis, J. H., Kiffe, T. R., 2002. On interacting bee/mite populations: a stochastic model with analysis using cumulant truncation. Enviromental and Ecological Statistics 9, 237-258.

Matis, J. H., Kiffe, T. R., Parthasarathy, P. R., 1998. On the cumulant of population size for the stochastic power law logisitc model. Theoretical Population Biology $53,16-29$.

Nåsell, I., 2001. Extinction and quasi-stationarity in the verhulst logistic model. J. of Theoretical Biology 211, 11-27.

Nåsell, I., 2003a. An extension of the moment closure method. Theoretical Population Biology 64, 233-239.

Nåsell, I., 2003b. Moment closure and the stochastic logistic model. Theoretical Population Biology 63, 159-168.

Pearl, R., Reed, L. J., 1920. On the rate of growth of the population of the united states since 1790 and its mathematical representation. Proceedings of the National Academy of Sciences U.S.A 6, 275-288.

Pielou, E. C., 1977. Mathematical Ecology. Wiley, New York.

Singh, A., Hespanha, J. P., 2006. Moment closure for the stochastic logis- 
tic model. Tech. Rep. CCEC-06-0117, Center for Control, Dynamical Systems and Computation. University of California at Santa Barbara, available at http://www. ece.ucsb.edu/ hespanha/techrep.html.

Verhulst, P. F., 1838. Notice sur la loi que la population suit dans son accroissement. Corr. Math. et Phys., 113-121.

Whittle, P., 1957. On the use of the normal approximation in the treatment of stochastic processes. J. Roy. Statist. Soc., Ser. B 19, 268-281. 